\title{
NUEVOS DATOS SOBRE STOMATOPODA Y DECAPODA, EXCEPTO ANOMURA (CRUSTACEA), DEL PARQUE NACIONAL DE COIBA, PACÍFICO DE PANAMÁ
}

\author{
M. Ortiz ${ }^{1}$, R. Lalana ${ }^{1}$, C. Varela ${ }^{1}$ y M. Capa ${ }^{2}$
}

\begin{abstract}
RESUMEN
Se presentan los resultados del estudio de las especies de decápodos (excepto Anomura) y estomatópodos recolectados en cuatro campañas científicas realizadas desde 1996 a 1998 al Parque Nacional de Coiba, Pacífico de Panamá. Se han identificado 35 especies de estos crustáceos, de las cuales 27 constituyen nuevos registros para dicho Parque. Se estima a partir de estos resultados que con un futuro muestreo más intenso se llegue a determinar un número superior de taxa para este interesante Parque Nacional.

Palabras clave: Crustacea, Decapoda, Stomatopoda, Pacífico, Panamá, Zoogeografía.
\end{abstract}

\section{ABSTRACT \\ New data on Stomatopoda and Decapoda, except Anomura (Crustacea) from Coiba National Park, Panamanian Pacific}

The decapod (except Anomura) and stomatopod crustaceans collected during four expeditions during 1996 and 1998 to the Parque Nacional de Coiba, in the pacific coast of Panamá, are herein presented. Twenty seven of the 35 species mentioned are new for the Coiba National Park. It is deduced from these results that intensive samplings in the future will reveal an higher number of taxa for this interesting Panamanian National Park. Key words: Crustacea, Decapoda, Stomatopoda, Pacific, Panamá, Zoogeography.

\section{Introducción}

Desde 1992 la Agencia Española de Cooperación Internacional ha estado colaborando con Panamá para desarrollar el Sistema de Parques Nacionales panameño, mediante el proyecto Planificación y Desarrollo de Parques Nacionales. Desde entonces el Instituto Nacional de Recursos Naturales Renovables, el Consejo Superior de Investigaciones Científicas y la Universidad
Nacional de Panamá, han dirigido y coordinado el estudio de la flora y la fauna terrestre, dulceacuícola y marina del Parque Nacional de Coiba, en el Pacífico de Panamá (entre las coordenadas $7^{\circ} 10^{\prime} 4^{\prime \prime}$, $7^{\circ} 53^{\prime} 27^{\prime \prime} \mathrm{N}$ y $81^{\circ} 32^{\prime} 35^{\prime \prime}$ y $81^{\circ} 56^{\prime} 15^{\prime \prime} \mathrm{O}$ ), junto a algunas instituciones y universidades españolas. El primero de los trabajos con estos resultados es un inventario preliminar de la flora y la fauna del mencionado Parque, editado por Castroviejo (1997). Los únicos antecedentes publicados sobre crustáce-

Centro de Investigaciones Marinas, Universidad de La Habana. La Habana, Cuba.

Laboratorio de Biología e Invertebrados Marinos, Departamento de Biología, Universidad Autónoma de Madrid, 28049 Madrid, España. 
os de este parque son el trabajo de San Martín y colaboradores (1997) con un listado de fauna y flora bentónica con 13 especies de crustáceos y la descripción de una nueva especie de anfípodo (Ortiz et al., 2000).

\section{Material y métodos}

El material para este trabajo fue recolectado por el equipo del Laboratorio de Invertebrados y Biología Marina de la Universidad Autónoma de Madrid, dentro del proyecto Flora y Fauna del Parque Nacional de Coiba, en cuatro campañas científicas que tuvieron lugar en junio de 1996, febrero de 1997, noviembre de 1997 y septiembre de 1998.

Se tomaron muestras cualitativas efectuadas en las ocho estaciones del listado que se presenta a continuación. Las muestras submareales se tomaron mediante buceo con escafandra autónoma.

El tipo de ambiente muestreado en las distintas estaciones aparece con un código con el siguiente significado: M, playa asociada a manglar; A, bloques de Pocillopora spp, tomados en arrecifes coralinos; IM, intermareal, bajo piedras; FC, Fondo coralino no arrecifal.

Lista de estaciones, tipo de ambiente muestreado y fechas de recolección:

A) Estación Biológica (IM) 30VI96, 13X97, 14 X97 y 29XI97; (M) 21VI96, 14XI97.

B) Granito de Oro (A) 14XI 97 y 19XI97.

C) Santa Cruz (M) 24VI96, 15XI97; (FC) 27VI96.

D) Canal de Afuera (A) 15XI95.

E) Rosario, playa (A) 16XI97.

F) Machete, playa (A) 18XI97.

G) María, ensenada (A) 23VI96, 21 XI97.

H) Jicarita, isla (FC) 22XI97 y (IM) 22 XI97.

La toponimia empleada ha sido la utilizada por Cardiel et al. (1997).

Para la identificación de las especies se han empleado los trabajos de Rathbun (1918, 1925, 1930), Kim y Abele (1976, 1988), Retamal (1981), Abele y Kim (1989), Hendrickx y Salgado Barragán (1991), Lemaitre y Álvarez León (1992), Hendrickx (1994, 1997, 1999), Hendrickx et al. (1997), Chace (1998) y Kamella et al. (1998).

Tanto las provincias zoogeográficas americanas, en las cuales hay representantes de los crustáceos de Coiba, como la validez de las especies de decápodos fueron revisadas según Boschi (2000). Los nombres en español de estas provincias son: Oregoniana (3), Californiana (4), de Cortéz (5), Panameña (6), de las Galápagos (7), de Perú-Chile (8), Magallánica (9), Argentiniana (10), Brasileña (11), Caribeña (12), Texana (13), Carolineana (14), Virginiana (15).

El asterisco $(*)$ después del autor y año de una especie, en el listado, significa un nuevo registro para el Parque Nacional de Coiba.

\section{Lista de especies}

Se ha hallado un total de dos especies de estomatópodos pertenecientes a dos géneros y dos familias distintas y 33 especies de decápodos pertenecientes a 25 géneros de 12 familias.

Clase Malacostraca Latreille, 1806 Subclase Hoplocarida Calman, 1904 Orden Stomatopoda Latreille, 1817 Familia Gonodactylidae Giesbrecht, 1910 Neogonodactylus cf. bredini (Manning, 1969) (12 y 14)

Superfamilia Lysiosquilloidea Giesbrecht, 1910

Familia Lysiosquillidae Giesbrecht, 1910 Lysiosquilla desausserii (Stimpson, 1857) * (6)

Subclase Eumalacostraca Grobben, 1892 Orden Decapoda Latreille, 1803

Familia Alpheidae Rafinesque, 1815 Alpheus rostratus Kim y Abele, 1988* (6 y 7) Alpheus panamensis Kingsley, 1878* (6 y 8) Alpheus normani Kingsley, $1878^{*}(5,6,7,10,11,12,13$, 14 y 15$)$

Alpheus sp.

Synalpheus sp.*

Familia Leucosidae Samouelle, 1819

Leucosilea jurinei (Saussure, 1853) * (6 y 7)

Familia Majidae Samouelle, 1819 Collodes sp.*

Subfamilia Inachinae McLeay, 1838

Mithrax sp.*

Subfamilia Majinae Samouelle, 1819

Ala cornuta (Stimpson, 1860) * (5 y 6)

Thoe sulcata panamensis Nobili, 1901*(6)

Familia Pisidae Dana, 1851

Herbstia campthacantha (Stimpson, 1860) * (6)

Familia Portunidae Rafinesque, 1815

Subfamilia Portuninae Rafinesque, 1815

Callinectes arcuatus Ordway, 1863 (4, 5, 6, 7, 8 y 12)

Familia Goneplacidae McLeay, 1838

Chasmophora macrophtalma (Rathbun, 1898) * (6) 
Familia Xanthidae McLeay, 1838

(Subfamilias omitidas)

Eurypanopeus crenatus (Milne Edwards y Lucas, 1843)* $(6,8$ у 9$)$

Eurypanopeus planus (Smith, 1869)* (6)

Eurypanopeus transversus (Stimpson, 1860) * (6, 7 y 8)

Panopeus chilensis Milne Edwards y Lucas, 1844* (6 y 8)

Panopeus sp.

Ozius perlatus Stimpson, $1859 *$ (6 y 7$)$

Pilumnus reticulatus Stimpson, $1860 *(6,10,11$ y 12)

Pilumnus sp.

Eriphia squamata Stimpson, 1859 (4, 5, 6, 7 y 8)

Trapezia ferruginea Latreille, $1825 *$ (6 y 7 )

Paractaea sulcata Stimpson, $1860 *$ (5, 6 y 7$)$

Xanthodius cooksoni (Miers, 1877)* (6 y 7)

Xanthidae no determinado

Familia Dairidae Ng \& Rodríguez, 1986

Daira americana Stimpson, 1860* (4, 6 y 7)

Familia Pinnotheridae De Haan, 1833

Parapinnixa sp.* (en el interior de tubos de terebélidos)

Familia Gecarcinidae McLeay, 1838

Gecarcinus quadratus Saussure, 1853 * (5, 6 y 12)

Familia Grapsidae McLeay, 1838

Subfamilia Grapsinae McLeay, 1838

Pachygrapsus transversus (Gibbes, 1850) * (3, 4, 6, 7, 8, $10,11,12,13,14)$

Subfamilia Plagusinae Dana, 1851

Plagusia inmaculata Lamarck,1818* (parasitada por un rizocéfalo) $(6,7$ y 8$)$

Familia Ocypodidae Rafinesque, 1815

Subfamilia Ocypodinae Rafinesque, 1815 Uca sp.

Ocypode gaudichaudi Milne-Edwards \& Lucas, 1843 * $(6,7$ y 8$)$

\section{Conclusiones}

De las 35 especies halladas, siete de las identificaciones se realizaron hasta nivel de género por el estado del material o por tratarse de juveniles. En tres de estos casos el género es un nuevo registro para el Parque Nacional de Coiba. De las 28 especies restantes 22 son nuevas citas para dicho Parque (79\% de los taxa). Una de las especies de estomatópodos sería una nueva cita para la provincia Panameña si se confirma su identificación. Del resto de las especies, cinco son endémicas de la provincia Panameña, otras cinco se encuentran también en la provincia de Galápagos y tres se distribuyen desde la provincia Panameña hasta la de Perú-Chile, pasando por las Galápagos. Una espe- cie se encuentra en las provincias de Cortez y Panameña y otra en estas dos y la provincia Californiana. Cinco especies son anfiamericanas, con distribuciones de mayor o menor amplitud en cada caso.

\section{AGRADECIMIENTOS}

Queremos expresarle nuestro agradecimiento a Guillermo San Martín y su equipo de la Universidad Autónoma de Madrid, por habernos cedido el material objeto de este estudio. Nuestro más sincero reconocimiento a los doctores Michael Hendrikx, Enrique Boschi y Rafael Lemaitre por el envío de literatura especializada, sin la cual la culminación exitosa de este trabajo no hubiera sido posible.

\section{Referencias}

Abele, G. \& KiM, W., 1989. The decapods crustaceans of the Panama Canal. Smithsonian Contributions to Zoology, 482: 1-50.

Boschi, E. E., 2000. Species of decapod crustaceans and their distribution in the American marine zoogeographic provinces. Revista de Investigación y Desarrollo Pesquero, 13: 7-136.

Cardiel, J. M., Castroviejo, S. \& Velayos, M., 1997. El Parque Nacional de Coiba: El Medio físico. In: S. Castroviejo (ed.). Flora y Fauna del Parque Nacional de Coiba (Panama). Inventario preliminar. AECI. Madrid: 11-30.

Castroviejo, S., 1997. Flora y Fauna del Parque Nacional de Coiba (Panamá). Inventario preliminar. AECI. Madrid. 534 pp.

Chace, F. A. JR., 1988. The caridean shrimps (Crustacea: Decapoda) of the Albatros Philippine Expedition, 1907-1910, part V: family Alpheidae. Smithsonian Contributions to Zoology, 466: 1-99.

Hendrickx, M. E., 1994. Catálogo de crustáceos decápodos y estomatópodos. Colección de referencia, Estación Mazatlán. CONABIO, ICML-UNAM. México D.F. 134 pp.

HENDRICKX, M. E., 1997. Los cangrejos braquiuros (Crustacea: Brachyura: Dromiidae hasta Leucosiidae) del Pacífico mexicano. CONABIO, ICML, UNAM. México D.F. 178 pp.

HENDRICKX, M. E., 1999. Los cangrejos braquiuros (Crustacea: Brachyura: Majoidea y Parthenopoidea) del Pacífico mexicano. CONABIO, ICML, UNAM. México D.F. 274 pp.

Hendrickx, M. E., Espinosa Pérez, M. Del C., Salgado Barragán, J. \& MÉndez Ubach, M. N., 1997. Tercer catálogo de la colección de referencia de invertebrados. CONABIO, ICML, UNAM. México D.F. 90 pp. 
Hendrickx, M. E. \& Salgado Barragán, J., 1991. Los estomatópodos (Crustacea: Hoplocarida) del Pacífico mexicano. Instituto de Ciencias del Mar y Limnología, Universidad Nacional Autónoma de México, Publicación Especial, 10: 1-200.

Kamella, A., Moscoso, B. V. \& Lleellish, M., 1998. Los crustáceos decápodos y estomatópodos del Perú. Informe del Instituto del Mar del Perú, 136: 80-109.

Lemaitre, R. \& Álvarez León, R., 1992. Crustáceos decápodos del Pacífico colombiano: Lista de especies y consideraciones zoogeográficas. Anales del Instituto de Investigaciones Marinas de Punta Betín, Santa Marta, Colombia, 21: 33-76.

Ortiz, M., Winfield, I. \& LALANA, R., 2001. Una nueva especie de anfípodo del género Bogidiella (Gammaridea, Bogidiellidae) de la Isla de Coiba, en el Pacífico de Panamá. Avicennia, 14: 47-52.

Rathbun, M. J., 1918. The grapsoid crabs of America. United State National Museum. Bulletin, 97: 1-461.

RATHBUN, M. J., 1925. The spider crabs of America. United State National Museum. Bulletin, 129: 1-613.

RATHBUN, M. J., 1930. The cancroid crabs of America of the families Euryalidae, Portunidae, Atelecyclidae, Cancridae and Xanthidae. United State National Museum. Bulletin, 152: 1-609.
Retamal, M. A., 1981. Catálogo ilustrado de los crustáceos decápodos de Chile. Gayana Zoología, 44: 136.

San Martín, G., López, E., Redondo, M., Capa, M, Cladera, P. \&. Laborda, J. A., 1997. El bentos marinos del Parque Nacional de Coiba (Panamá). En: S. Castroviejo (ed.). Flora y Fauna del Parque Nacional de Coiba (Panama). Inventario preliminar. AECI. Madrid: 35-55. 